\title{
China's Service Trade Competitiveness: A Study based on Value-added Trade
}

\author{
Yi Cao \\ Department of International Economics and Business, Xiamen University, Xiamen City, P. R. China
}

\begin{abstract}
The competitiveness of China's service trade has long been focused. Researchers often use different indices to estimate it. However the characteristics of trade changed a lot due to the production segmentation. Intermediate goods become the main part of trade and lead to double counting problems of trade statistics. The indices established on trade statistics probably biased. Therefore, this paper uses MRIO model and WIOD database to calculate China's value-added trade from 1995 to 2009, which excluded the double counting part, and then re-estimates RCA index to analysis China's service trade competitiveness in the global value chain. Results find that trade statistics underestimate China's RCA of service trade. The Transportation and Hotels and Restaurants are more competitive while the high value-added industries, such as Financial Intermediation and Post and Telecommunication are less competitive.
\end{abstract}

Keywords - service trade competitiveness; value-added trade; multi-regional input-output model

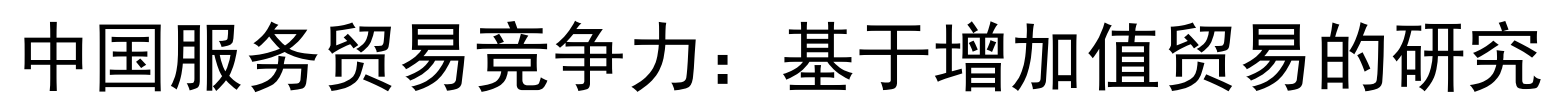

曹毅

厦门大学国际经济与贸易系, 厦门, 福建, 中国

摘 要 服务贸易的竞争力问题一直是学界研究的焦点和政策制定者关注的重点, 不少学者通过测算一系列指标评价中国服务贸 易的竞争力。然而当今国际贸易的特点发生了深刻的变化, 以跨国公司为主导的全球价值链进一步深化使得中间品成为贸易的主体, 使得贸易统计数据存在重复计算的问题，因而基于贸易统计数据测算的一系列评价指标很可能有所偏误。基于此本文从增加值贸易的 角度出发, 运用多区域投入产出模型和世界投入产出数据库, 测算了 1995-2009 年中国服务贸易整体和分部门的 RCA 指数。实证结果 发现运用贸易统计数据低估了中国服务贸易的 RCA 指数。中国服务贸易整体有一定的竞争力, 但是主要以资源和劳动密集型的运输 业和餐饮旅游业带动, 而附加值较高的金融业、通信业等竞争力较弱。

关键词 服务贸易竞争力, 增加值贸易, 多区域投入产出模型

\section{1. 引言}

通讯和信息技术的进步，一方面使得国际分工进一步 细化, 由生产与消费的分割演化为当前生产与生产分割的 贸易格局(Baldwin, 2006), 形成了以全球价值链为主导的当 代国际贸易模式; 另一方面也带动了传统的运输服务业以 及信息和通讯等新兴服务业的发展。

中国作为世界加工厂, 积极参与全球价值链。近年来 中国的服务业虽然迅速发展, 但是服务贸易增长速度不及 服务业发展的速度。服务贸易总量逐年上升, 但进口大于 出口, 并主要从事旅游、运输等传统服务业, 属于资源和 劳动力密集型的行业; 而知识和资本密集型的新兴服务业
附加值高, 其占服务贸易总出口的比重较小。因而无论从 数量还是质量上来看, 我国的服务贸易竞争力还较弱。服 务贸易竞争力问题一直以来都是学界和政策制定者关注的 一大焦点。

显示性比较优势指数(RCA)作为刻画一国竞争力大小 的重要指标, 在对服务贸易竞争力的实证研究中也被广泛 应用。但是已有研究所选用的数据基础都涉及到贸易统计 数据, 即进出口额。但是值得注意的是, 中间品贸易的盛 行使得传统贸易统计数据严重的重复计算问题。世贸组织 总干事 Pascal Lamy 在日内瓦提出, 传统国际贸易统计侧重 于计算进出口总额, 应以进出口贸易中的各国国内增加值 
作为贸易统计的标准。我国以加工贸易为主, 从而中间品 进出口占比重较大, 利用贸易进出口额测算的指标和变量 的选取显然会由于重复计算问题而导致实证结果与客观事 实存在一定的偏误。因此本文认为有必要从增加值贸易的 角度对我国服务贸易的竞争力进行研究。一来避免了贸易 统计数据的重复计算问题所带来的偏误, 更加客观地刻画 我国服务贸易竞争力的现状, 另一方面也为提高我国服务 贸易竞争力提供一些切实可行的有针对性的政策建议。

本文在第二部分对有关服务贸易竞争力和测算增加值 贸易的文献进行回顾, 第三部分介绍将贸易总额转化为增 加值贸易的模型和数据处理, 第四部分呈现本文的实证结 果和分析, 最后一部分进行总结并提出一些提高我国服务 贸易竞争力的政策建议。

\section{2. 文献综述}

\section{1 对服务贸易竞争力的理论与实证研究}

大部分学者认为贸易产生的动因在某种程度上等同于 贸易竞争力的来源, 并在 H-O 理论框架和新贸易理论的框 架下证明了比较优势和规模经济的理论一定程度上适用于 服务贸易。90 年代之前, 不少西方学者普遍证实了比较优 势理论对服务贸易仍然适用(如 Deardorff, 1985), 即各国劳 动生产率和资源要素禀赋的差异是服务贸易产生的动因, 也是服务贸易竞争力的直接来源。之后, 随着产业内贸易 的盛行, 新贸易理论认为即使没有要素禀赋的比较优势, 规模经济、产品差异化也能促进国际贸易的发展。服务产 品正是一种差异化极大的产品, 在此框架下, 不少学者通 过实证研究证明了国家间的相似程度 (如收入水平和需求 的相似性) 是服务贸易产生的动因从而成为服务贸易竞争 力的来源。

对服务贸易竞争力的实证研究主要有两类。一类基于 比较优势的理论基础, 对一系列显示性指标进行测算从而 评价一国的服务贸易的竞争力。Hoekman 和 Karsenty(1992) 运用美国经济学家 Balassa 提出的显示性比较优势指数 (RCA) 分析了不同收入水平的国家在服务贸易上的比较优 势。国内也有不少学者运用 RCA 指数来测算我国的服务贸 易比较优势 (如陈虹和章国荣, 2010 ), 大部分结果表明, 我 国服务贸易的国际竞争力较弱, 且有下降的趋势。除了以 上两种常用的指标外, 还有不少学者结合其他指标, 如比 较出口业绩指标(CEP)、贸易重叠指标(TO) 和出口相似指标 (ES)等来综合评价一国的服务贸易竞争力(如 Bobirca 和 Miclaus, 2007)。另一类则主要以波特的 “钻石模型” 为理 论框架, 运用计量模型对影响贸易竞争力的 4 组基本决定 因素和 2 种辅助因素进行回归检验, 分析影响贸易竞争力
的因素。如以人口结构素质、货物贸易出口总额、服务业 劳动生产率、城市化水平和第一产业劳动生产率等作为相 应因素的解释变量。部分结果表明, 人均收入、服务贸易 开放度、服务业发展水平等对中国服务贸易竞争力具有显 著促进作用(陈虹和章国荣, 2010)。

\section{2 全球价值链视角下的国际贸易}

为了避免多重计算问题, 刻画当今国际贸易的新特征, OECD 和 WTO(2010) 均提出了 “增加值贸易 (trade in value-added)" 的概念, 将其与贸易统计数据作为互补的数 据。值得注意的是, 增加值贸易不只是一种统计或计算上 的概念, 实际上它也有相应的理论基础。Grossman 和 Rossi-Hansberg(2008)提出以 “任务的贸易(trade in tasks)” 来模型化当今全球价值链推动的国际贸易。他们将生产过 程概念化为许多任务(tasks), 1 单位某种产品的生产需要通 过一系列的任务来实现, 每种任务的完成需要一些生产要 素来实现(如低技术劳动力、高技术劳动力和资本等)。一方 面, 对于技术、要素禀赋和相对要素价格不同的国家来说, 这个模型就类似于新古典贸易理论中的要素比例模型, 其 不同之处在于这个模型用刻画完成某种任务的技术的生产 函数代替原来的体现商品生产技术的生产函数; 另一方面, 对于拥有相似技术和相似要素禀赋的国家来说, 规模经济 是其价值链分割的主要原因(Grossman 和 Rossi-Hansberg, 2012)，类似于新贸易理论。

Johnson 和 Noguera(2012)在 Hummels et al.,(2001)首次 尝试使用一国投入产出表测算垂直专业化的程度 (即一国 出口中的进口份额)的基础之上, 对增加值出口做出了正式 的定义: 由一个国家生产, 但被其他国家吸收的增加值, 并提出了总出口中的增加值(VAX)比率的概念。Koopman et al., (2010)运用多区域投入产出模型(MRIO)把出口完全分 解为 5 个增加值部分之和, 不同部分的组合可以得到几乎 之前所有学者的指标, 并能够明确计算出 “重复计算” 部 分。在此框架下, 他们测算了各部门参与国际生产链的程 度以及在国际生产链中的位置重新测算了显示性比较优势 (RCA) 指数, 发现某些国家某些部门的 RCA 指数与用贸易 统计数据计算的结果有较大差异。

\section{3. 模型和数据}

\section{1 模型的构建}

首先构建一个 $n$ 国 $r$ 部门的多区域投入产出模型。令: 


$$
\begin{gathered}
A=\left(\begin{array}{cccc}
A_{11} & A_{12} & \cdots & A_{1 n} \\
A_{21} & A_{22} & \cdots & A_{2 n} \\
\vdots & \vdots & \ddots & \vdots \\
A_{n 1} & A_{n 2} & \cdots & A_{n n}
\end{array}\right)^{-1} \\
\boldsymbol{B}=(\boldsymbol{I}-\boldsymbol{A})^{-1}=\left(\begin{array}{cccc}
\boldsymbol{B}_{11} & \boldsymbol{B}_{12} & \cdots & \boldsymbol{B}_{1 n} \\
\boldsymbol{B}_{21} & \boldsymbol{B}_{22} & \cdots & \boldsymbol{B}_{2 n} \\
\vdots & \vdots & \ddots & \vdots \\
\boldsymbol{B}_{n 1} & \boldsymbol{B}_{n 2} & \cdots & \boldsymbol{B}_{n n}
\end{array}\right)
\end{gathered}
$$

其中, 对角线上的分块矩阵 $\boldsymbol{A}_{i i}$ 表示 $i$ 国的国内中间消 耗系数矩阵, 其元素 $a_{m z}^{i i}$ 表示 $i$ 国生产 1 单位 $z$ 产品需要本 国的 $m$ 部门的中间投入量。非对角线上的分块矩阵 $\boldsymbol{A}_{i j}$ 表示 $j$ 国生产过程中需要 $i$ 国的中间投入, 其元素 $a_{m z}^{i j}$ 表示 $j$ 国 生产过程 1 单位 $z$ 产品需要 $i$ 国的 $m$ 部门的中间投入量。 $\boldsymbol{B}$ 是 MRIO 模型的里昂惕夫逆矩阵, 其中的元素与 $\boldsymbol{A}$ 相同的 位置表示相应的完全投入量。由 Balassa(1975)提出的显示 性比较优势指数(RCA)的计算公式如(3)所示:

$$
R C A_{i j}=\frac{e x_{i j} / \sum_{j} e x_{i j}}{\sum_{i} e x_{i j} / \sum_{i} \sum_{j} e x_{i j}}
$$

其中, $R C A_{i j}$ 表示 $i$ 国 $j$ 部门的显示性比较优势指数, $e x_{i j}$ 是 $i$ 国 $j$ 部门的出口额, $\sum_{j} e x_{i j}$ 表示 $i$ 国的总出口; $\sum_{i} e x_{i j}$ 表示全球 $j$ 部门产品的总出口, $\sum_{i} \sum_{j} e x_{i j}$ 是全球的 总出口额。由于进出口统计数据存在重复计算问题, 因此 我们运用 Koopman et al.,(2010)的框架将出口完全分解为各 国增加值之和, 用出口中的本国增加值来代替出口。令 $d v_{i j}$ 表示出口中的本国增加值, 则有:

$$
R C A_{i j}=\frac{d v_{i j} / \sum_{j} d v_{i j}}{\sum_{i} d v_{i j} / \sum_{i} \sum_{j} d v_{i j}}
$$

$d v_{i j}$ 可以由多区域投入产出模型测算而得。用 $\boldsymbol{V}$ 表示 增加值系数矩阵, $\boldsymbol{B}$ 表示里昂惕夫逆矩阵, $\boldsymbol{E}$ 表示出口向 量, 则有:

$$
D V=V B E=\left(\begin{array}{cccc}
V_{1} B_{11} E_{1} & V_{1} B_{12} E_{2} & \cdots & V_{1} B_{1 n} E_{n} \\
V_{2} B_{21} E_{1} & V_{2} B_{22} E_{2} & \cdots & V_{2} B_{22} E_{n} \\
\vdots & \vdots & \ddots & \vdots \\
V_{n} B_{n 1} E_{1} & V_{n} B_{n 2} E_{2} & \cdots & V_{n} B_{n 2} E_{n}
\end{array}\right)
$$

则由(3)式可得, 出口被完全分解成了增加值相加的形 式。其中, 对角线上的元素表示一国出口中的国内增加值

$$
D V_{r}=V_{r} B_{r r} E_{r}
$$

$d v_{i j}$ 则是出口中的国内增加值中的 $i$ 行 $j$ 列元素。结合 (6)式和(4)式则可以得到基于出口中的增加值所测算的 RCA 指数。

\section{2 数据来源和处理}

目前国际上最新的多区域投入产出数据库主要包括根 据 OECD 投入产出数据库建立的 GRAM 数据库、根据 GTAP 建立的完整的 GMRIO 数据库、发展中经济体研究所 -日本对外贸易组织(Institute of Developing Economies, Japan External Trade Organization, IDE-JETRO)构建的亚洲 国际投入产出表(Asian International IOTs, AIIOTs), 以及后 来发展的多部门多国家的 EORA 数据库, 提供与环境相关 的信息的 EXIOPOL 数据库(简称为 EXIOBASE), 以及仅使 用公开可得数据构建的世界投入产出数据库 (world input-output database, WIOD)。

WIOD 数据库囊括了全世界所有国家和地区(40 个国 家和 1 个世界其他国家), 在时间维度上拥有 1995-2009 年 连续的数据, 并且每年的数据都提供了现价表和以上一年 价格为不变价格的 MRIO 表(1995 年除外)。从部门分类来 看, WIOD 的 MRIO 表涵盖了 35 个部门, 其中服务部门有 17 个(如表 2 所示)为我们的分析提供了良好的数据基础。 因此本文选择 WIOD 数据库提供的多区域投入产出表。为 了消除价格的影响, 我们将 1995-2009 年的 15 张 MRIO 表 平减为 2002 年的可比价格。

\section{4. 实证结果分析}

表 1 显示了 1995、2000、2005 和 2009 年我国服务贸 易的 RCA 指数。一般而言, 若 RCA 指数大于 2.5 , 则表明 该国类产品具有极强的国际竞争力; 若介于 2.5-1.25 之间 则认为该国某产品具有很强的国际竞争力; 若介于 1.25-0.8 之间则表明该国某类产品具有较强的国际竞争力; 而如果 $\mathrm{RCA}$ 指数小于 0.8 则说明认为该国该类产品的国际竞争力 较弱。

总体来看, 中国服务贸易的 RCA 指数在这 4 个年度分 别为 $0.8 、 0.98 、 0.85$ 和 1.10 。相对于以往基于贸易统计数 据的研究而言, 服务贸易整体的 RCA 指数提高了 (如陈宪 和殷风(2008)测算出 2005 年我国服务贸易的 RCA 指数仅 为 0.4658 ; 何骏和郭岗(2013)测算的 2009 年中国服务贸易 $\mathrm{RCA}$ 指数仅为 0.34 )。我国的服务贸易整体来看还是有一定 的国际竞争力的。

从部门的情况来看, 酒店餐饮业和运输业的显示出较 强的竞争力。而运输业中的水上运输业的比较优势较为突 出, 这主要是由于国际贸易的迅速发展带动了运输业特别 
表 1 基于增加值出口测算的中国服务贸易的 RCA 指数

\begin{tabular}{|l|l|l|l|l|}
\hline \multicolumn{1}{|c|}{ 部门/年份 } & 1995 & 2000 & 2005 & 2009 \\
\hline 建筑业 & 0.65 & 0.39 & 0.23 & 0.26 \\
\hline 商品批发、代理销售业 & 1.14 & 1.24 & 0.37 & 1.27 \\
\hline 商品零售业 & 0.51 & 0.62 & 0.68 & 0.73 \\
\hline 酒店餐饮业 & 2.81 & 1.71 & 1.48 & 1.47 \\
\hline 陆地运输业 & 1.69 & 1.36 & 1.13 & 1.17 \\
\hline 水上运输业 & 1.06 & 1.95 & 2.25 & 1.91 \\
\hline 航空运输业 & 1.57 & 1.06 & 1.08 & 0.90 \\
\hline 其他辅助性运输活动 & 1.35 & 0.43 & 0.35 & 0.39 \\
\hline 邮政通信业 & 0.99 & 0.97 & 0.87 & 0.80 \\
\hline 金融业 & 0.91 & 0.66 & 0.51 & 0.61 \\
\hline 房地产业 & 0.92 & 0.40 & 0.36 & 0.52 \\
\hline 设备租赁及其他商业服务业 & 0.40 & 0.33 & 0.33 & 0.36 \\
\hline 公共管理、国防及社会保障业 & 0.16 & 0.07 & 0.07 & 0.22 \\
\hline 教育 & 1.31 & 0.63 & 0.65 & 0.88 \\
\hline 医疗卫生和社会工作 & 0.94 & 0.72 & 1.30 & 1.51 \\
\hline 其他社会、社区和个人服务 & 1.85 & 1.36 & 0.86 & 0.87 \\
\hline
\end{tabular}

是水上运输业的发展。邮政通信业的现实性比较优势在 0.90 左右, 显示出较强的竞争力。而像金融业、设备租赁 及其他商业服务业这种附加值较高的服务部门的 RCA 指 数较低, 竞争力较弱。因此可以看出, 我国服务贸易的总 体竞争力水平虽然不高, 但逐年上升。

但是从结构上来看, 竞争力主要是依靠资源密集型和劳 动力密集型的行业所带动的, 而资本和技术密集型的行业 竞争力仍然较弱, 甚至有下降的趋势。

\section{5. 总结}

本文在全球价值链的视角下, 基于增加值贸易的概念 对中国服务贸易的 RCA 指数进行重新测算, 避免了贸易统 计数据存在的重复计算问题, 更加客观地刻画了全球价值 链下中国服务贸易的竞争力。实证结果发现, 中国服务贸 易整体 RCA 指数在 0.9-1.0 左右。说明以贸易统计数据为 基础测算的中国服务贸易的 RCA 指数中国服务贸易的竞 争力。虽然本文结果显示出中国服务贸易具有一定的竞争 力, 但是从部门分布来看, 主要靠资源和劳动力密集型的 行业拉动, 附加值高的资本和技术密集型行业, 如金融业 的竞争力仍然较弱。

在对服务贸易竞争力问题研究上, 本文仅针对中国服 务贸易的 RCA 指数进行评估, 另外还有其他一些指标可以 用于综合评价中国服务贸易的竞争力。另外, 本文尚未分 析影响服务贸易竞争力的原因。因此进一步的研究方向可 以根据波特的 “钻石模型” 进行计量分析, 用增加值贸易
代替与贸易额有关的变量, 以期更加客观地分析影响我国 服务贸易竞争力的因素, 为政策制定者提供一定的参考。

\section{参考文献(References)}

[1] R. E. Baldwin, "Globalization: The great unbundling(s). In globalization challenges for Europe," Helsinki: Office of the Prime Minister of Finland, 2006.

[2] A. Bobirca, and P-G. Miclaus, "A Multilevel Comparative Assessment Approach to International Services Trade Competitiveness: The Case of Romania and Bulgaria," World Academy of Science, Engineering and Technology, vol. 6, pp.1-6, 2007.

[3] B. Balassa, "Trade Liberalization and Revealed Comparative Advantage," The Manchester School of Economic and Social Studies, vol.33, no.2, pp. 99-123, 1965.

[4] A. V. Deardorff, Major recent developments in international trade theory. Institute of Public Policy Studies, University of Michigan, 1985.

[5] G. M. Grossman and E. Rossi-Hansberg, "Trading Tasks: A Simple Theory of Offshoring," American Economic Review, vol. 98,no.5, pp.1978-1997, 2008

[6] B. Hoekman and G. Karsenty, "Economic Development and International Transaction in Services," Development Policy Review, vol.10, pp.35-67,1992

[7] D. Hummels, J. Ishii and K. Yi, "The Nature and Growth of Vertical Specialization in World Trade," Journal of International Economics, vol.54, pp.75-96, 2001.

[8] Johnson, R. C., and G. Noguera, “Accounting for Intermediates: Production Sharing and Trade in Value Added," Journal of International Economics, vol.86, no.2, pp.224-236, 2012.

[9] R., Koopman, W. Powers, Z. Wang and S.-J.Wei, "Give Credit Where Credit is Due: Tracing Value Added in Global Production Chains," NBER Working Paper 16426, 2010.

[10] H. Chen and G. R. Zhang, "The empirical study on China's service trade competitiveness," Management World, vol.10, pp.13-23, 2010.

[11] X. Chen and F. Yin, "Service trade: International characteristics and China's competitiveness," Finance and Trade Economics, vol.1, pp.113-128, 2008.

[12] J. He and L. Guo, "Study of the Upgrade of the Competitive Power of Service Trade in China-Based on the Empirical Analysis of Global Main Service Trade Countries Panel- Data”, Journal of Shanxi Finance and Economics University, vol.3, pp.44-55, 2013. 